\title{
Sox4 Up-Regulates Cyr61 Expression in Colon Cancer Cells
}

\author{
Gang Wu Yuan-Zeng Zhu Jian-Cheng Zhang
}

Department of General Surgery, Henan Provincial People's Hospital, People's Hospital of Zhengzhou University, Zhengzhou, People's Republic of China

\section{Key Words}

Colon cancer • Sox4 $•$ Cyr61 $\bullet$ Gene regulation

\begin{abstract}
Background/Aims: Genetic changes leading to aberrant activation of oncogenes are viewed as a crucial step in colon cancer. Sox4, a member of Sox (Sry-box) family of transcription factors, plays a critical role in tumorigenesis. Methods: PCR-based microarrays were used to identify potential transcriptional target of Sox4. siRNA was used to knockdown the expression of Sox4. Luciferase and chromatin immunoprecipitation (ChIP) assays were used to test the transcriptional regulations. Results: PCR-based microarrays found that Cyr61, a secreted extracellular matrix-associated signaling protein, was a transcriptional target of Sox4. Overexpression of Sox4 increased, while its knockdown using small interfering RNA (siRNA) reduced Cyr61 expression. A potential Sox4 binding motif located at the proximal Cyr61 promoter was identified. Conclusion: Thus, our results suggest a previously unknown Sox4-Cyr61 molecular network, which may control colon cancer cell proliferation and survival.
\end{abstract}

\section{Introduction}

Colon cancer has become one of the most common malignant cancers worldwide, leading to half a million deaths each year [1]. Numerous reports have confirmed that multiple genetic changes that are critical for colon cancer initiation and progression, such as the Wnt/ $\beta$-Catenin pathway, Ras/Raf pathway and p53 pathway [2,3]. 
Sox4 is a 47-kDa protein, which belongs to the sex-determining region Y (SRY) box family, consisting of 20 highly conserved transcription factors [4]. Initial studies have demonstrated that Sox 4 is required for the development of several tissues or organs, including heart, lymphocytes, and thymocytes [5]. Indeed, ablation of Sox4 in mice resulted in cardiac defects and reduced proliferative capacity of B-cell progenitors [6, 7]. Subsequent studies in recent years suggested that Sox 4 might be an important oncogene contributing to tumor progression [8]. For instance, its expression was dramatically elevated in tumors of the prostate, bladder, breast and lung [9-11]. Besides, nuclear Sox4 expression was also increased in colon tumor tissues relative to non-tumor colon tissues [12]. Moreover, its overexpression was closely correlated with tumor invasion and metastasis [12], suggesting its potential significance in the clinical and prognostic implications. However, until now, the molecular mechanism by which Sox 4 exerts its activity in tumor progression and aggressiveness remains poorly understood.

In the current study, we performed a PCR-based microarrays using cDNA prepared from Sox4-overexpressed SW480 cells. Furthermore, Cyr61, a secreted extracellular matrixassociated signaling protein, was identified as a novel transcriptional target of Sox4 Our results suggest that the oncogenic roles of Sox 4 in colon cancer cells might be, at least in part, dependent on its up-regulation of Cyr61.

\section{Material and Methods}

\section{Tissue Samples}

20 pairs of human colon cancer tissues, adjacent non-cancerous normal tissues were collected from routine therapeutic surgery at our department. All samples were obtained with informed consent and approved by Henan Provincial People's Hospital review board.

\section{Cell Culture}

Two colon cancer cell lines (SW480 and HCT116) were purchased from Cell Bank of Type Culture Collection of Chinese Academy of Sciences (CAS, Shanghai, China) and cultured in modified Eagle's medium (MEM, Gibco, Shanghai) supplemented with $10 \%$ fetal calf serum, $100 \mathrm{IU} / \mathrm{ml}$ penicillin and $100 \mathrm{mg} / \mathrm{ml}$ streptomycin (Gibco, Shanghai).

\section{RNA extraction, Microarrays and Real-time Analysis}

Total RNAs were isolated from tissues or cells by TRIzol reagent, and reverse transcriptions were performed by Takara RNA PCR kit (Takara, Dalian, China) following the manufacturer's instructions. Affymetrix array hybridization and scanning were performed using Human Genome U133A 2.0 chips by Invitrogen Company (Shanghai, China). In order to quantify the transcripts of the interest genes, realtime PCR was performed using a SYBR Green Premix Ex Taq (Takara, Japan) on Light Cycler 480 (Roche, Switzerland). $\beta$-actin gene was used as an internal control. The primer sequences were listed as following: Cyr61 (Sense: 5'- ACCGCTCTGAAGGGGATCT-3'; Antisense: 5'- ACTGATGTTTACAGTTGGGCTG -3'); Sox4 (Sense: 5'-GACCTGCTCGACCTGAACC-3'; Antisense: 5'- CCGGGCTCGAAGTTAAAATCC-3'); $\beta$-actin (Sense: 5'CTCGACACCAGGGCGTTATG-3'; Antisense: 5'- CCACTCCATGCTCGATAGGAT-3').

\section{Small interfering RNA, Transient Transfections and Luciferase assays}

Cells were plated on to six-well plates grown to $50 \sim 70 \%$ confluences and then transfected with 60 nM siGENOME non-targeting siRNA (5'-CAUGAUCCAUGGAUCGGAUU-3'), human Sox4 (Sox4-1: 5'- AUCUGAAUUGGUAcUGGAUAAU-3';Sox4-2:UCGAGCUUCCCCTAUCAUGG-3') or Cyr61 (5'GGUCAAAGUUACCGGGCAGU-3')siGENOME SMART pool (Millipore, USA). Human Cyr61 promoter was cloned into PGL4 plasmid (Promega, USA). All the transient transfections were performed by Lipofectamine 2000 (Invitrogen), according to the manufacturer's instructions. For luciferase reporter assay, SW480 cells were seeded in 24-well plates and transfected with the indicated plasmids. Luciferase activity was measured using the Dual Luciferase Reporter Assay System (Promega, USA). 
Wu/Zhu/Zhang: Sox4 Up-Regulates Cyr61

Western Blot

Cells were harvested and lysed with ice-cold lysis buffer (50 mM Tris- $\mathrm{HCl}, \mathrm{pH}$ 6.8, $100 \mathrm{mM}$ 2-ME, $2 \% \mathrm{w} / \mathrm{v}$ SDS, $10 \%$ glycerol). Proteins were separated by $10 \%$ SDS PAGE and transferred to NC membrane (Amersham Bioscience, Buckinghamshire, U.K.). After blocking with 10\% nonfat milk, membranes were immunoblotted with primary antibodies, followed by HRP-linked secondary antibodies (Santa Cruz, USA). The signals were detected by SuperSignal West Pico Chemiluminescent Substrate kit (Pierce, Rockford) according to manufacturer's protocols. Anti-Sox4, Cyr61, CK2, P65, AKT and acetylated histone H3 antibodies were purchased from Cell signaling Company (USA). Protein levels were normalized to $\beta$-actin (Abcam, USA).

\section{Chromatin Immunoprecipitation Assays}

A chromatin immunoprecipitation (ChIP) assay kit was used (Upstate, USA). In short, SW480 cells were fixed with formaldehyde for 15 min. DNA was sheared to fragments at 200-1000 bp by several sonications. The chromatin were incubated and precipitated with antibodies against Sox 4 antibody or IgG (Santa Cruz,USA). The primer sequences for the Cyr61 promoter regions were listed as following: -300 bp -100 bp (Forward: 5'-CAGCTTATGCATACGATACT-3', Reverse: 5'-ATCGTAAGCATACC TGCAAG-3'); -2000 bp -1800 bp (Forward: 5'-GGCATAGATCATTGCGGTA CT-3', Reverse: 5'-AATAGACTCACTAGCATGGA CT-3').

\section{BrdU Incorporation Assays}

A cell proliferation enzyme-linked immunosorbent assay kit (Beyotime, Shanghai, China) was employed to analyze the incorporation of BrdU during DNA synthesis following the manufacturer's protocols.

Tumor growth assay

Male BALB/c nude mice aged 6 weeks were purchased from the Experimental Animal Center of the Third Military Medical University. 6×105 SW480 cells stably expressing Sox4 or empty vector were injected subcutaneously to the skin under the front legs of the mouse. The mice were observed over 4 weeks for tumor formation. After the mice were sacrificed, the tumors were recovered and the wet weights of each tumor were determined. The experiments were performed using five mice per group.

\section{Statistical Analysis}

Values were shown as mean \pm SEM. Two-tailed Student's $t$ tests were used for two-group comparisons. A two-way analysis of variance (ANOVA) with Bonferroni-adjusted post tests were used for comparisons of more than two groups. GraphPad Prism 5.0 Software was employed to perform statistical analysis. Significance is displayed as * $(\mathrm{P}<0.05)$, ** $(\mathrm{P}<0.01)$ or $* * *(\mathrm{P}<0.001)$.

\section{Results}

\section{Identification of Cyr61 as a novel Sox4 responsive gene}

To screen potential transcriptional target of Sox 4 in colon cancer, SW480 cells were transfected with Sox 4 expression plasmids or empty vector for $24 \mathrm{hr}$. The clustering analysis of Affymetrix gene arrays revealed that 86 genes were significantly up-regulated in SW480 cells with Sox4 overexpression $(\mathrm{p}<0.05)$ (Data not shown). Based on this result, the gene with the largest difference in expression between two groups was Cyr61 (Fig. 1A). We further confirmed the up-regulation of Cyr61 mRNA and protein levels by ways of Real-time PCR and Western blot (Fig. 1B-1C). Protein levels of CK2, a downstream target gene of Sox4, were also determined by western blot. Besides, the induction of Cyr61 by Sox 4 was also observed from HCT116 cells (Fig. 1D-1E). Interestingly, Cyr61 expression was not changed by Sox 2 or Sox 9 overexpression (Data not shown), suggesting the specific roles of Sox 4 in the up-regulation of Cyr61.

Moreover, targeted gene knockdown of endogenous Sox4 using two different small interfering RNA (siRNA) also inhibited Cyr61 expression in both cells (Fig. 2A-2D), suggesting that Sox4 might be a transcriptional activator of the Cyr61 gene. 
Fig. 1. Identification of Cyr61 as a novel Sox4 target gene in colon cancer cells. (A) Cluster analysis of hepatic gene expression in SW480 cells transfected with Sox4 expression plasmids or empty vector (EV) for $24 \mathrm{hr}(\mathrm{n}=3)$. Then cells were harvested for RNA extraction and genes expression was analyzed by Affymetrix array hybridization and scanning. Data sets were statistically analyzed using Student's test. (B-E) mRNA and protein levels of Cyr61 were analyzed by Real-time PCR (B, D) and Western blot (C, E) in SW480 or HCT116 cells transfected with Sox4 expression plasmids or empty vector (EV) for 24 or 36 hr, respectively. $* * * \mathrm{p}<0.001$.

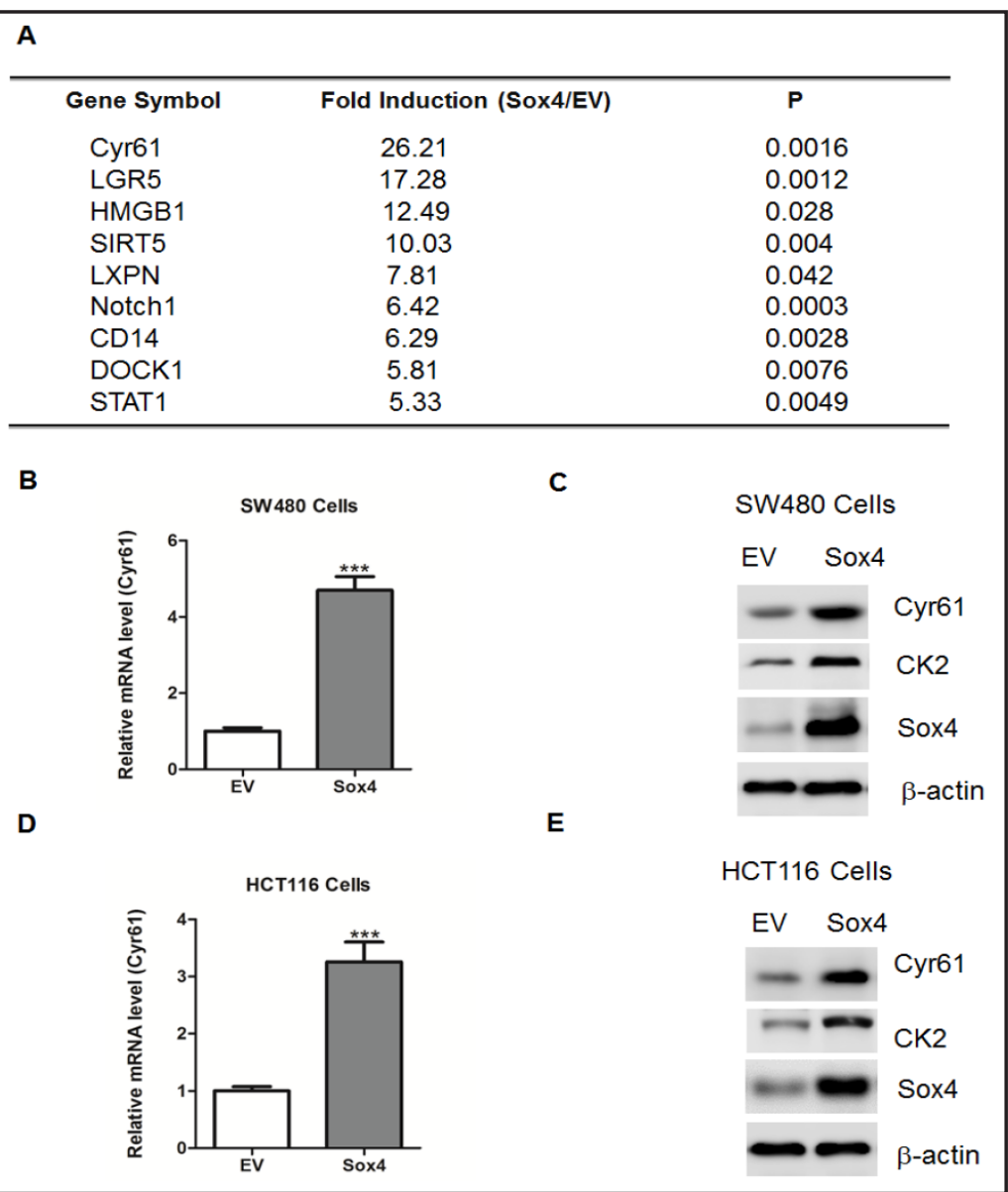

Sox4 regulates down-stream signaling pathway of Cyr61

Previous studies have demonstrated that Cyr61 promotes cell proliferation and survival through activation of Akt and NF-kB/P65 signaling [13, 14]. Therefore, we examined the downstream signaling pathways in colon cancer cells with Sox 4 overexpression or knockdown. As shown in Figure 3A and 3B, forced overexpression of Sox4 led to the activation of Akt and P65 in SW480 and HCT116 cells, as shown by increased phosphorylated AKT and P65 (Fig. 3A-3B), while its knockdown led to a decreased activation of Akt and P65 signaling (Fig. 3C-3D). Consistently, Sox 4 overexpression dramatically enhanced cell proliferative capacity in colon cancer cells, which was significantly abolished by Cyr61 deficiency (Fig. 3E-3F). Taken together, our results suggest that the up-regulation of Cyr61 represents an important mechanism for the Sox4-induced cell proliferation.

Sox4 regulates Cyr61 expression in vivo

Next, we generated SW480 cells with stable overexpression of Sox4 or empty vector (EV) for tumorigenic potential. Cells were then injected subcutaneously to the skin under the front legs of the nude mice. The tumor growth was closely monitored for another 4 weeks. As a result, the tumor size and weight was markedly increased in Sox4-overexpressed tumors compared to control tumors (Fig. 4A-4B), suggesting that Sox 4 could promote colon cancer growth in vivo. In addition, mRNA and protein levels of Cyr61 were also up-regulated by Sox 4 overexpression (Fig. 4C-4D)

Sox4 regulates Cyr61 gene transcription through promoter occupancy

Next, to seek the molecular basis for this regulation, human Cyr61 promoter region was scanned using TESS software [15]. As shown in Figure 5A, a potential Sox4 binding site was defined in the proximal region of Cyr61 promoter, located at the $-168 \mathrm{bp} \sim-162$ bp relative to 
Fig. 2. Sox 4 knockdown results in a reduction of Cyr61 expression. (AD) mRNA and protein levels of Cyr61 were analyzed by Real-time $\operatorname{PCR}(A, C)$ and Western blot $(B, D)$ in SW480 or HCT116 cells transfected with two siRNA oligos targeting Sox4 (Sox4-1, Sox4-2) or negative controls (NC) for 24 or $36 \mathrm{hr}$, respectively. $* * \mathrm{p}<0.01$.

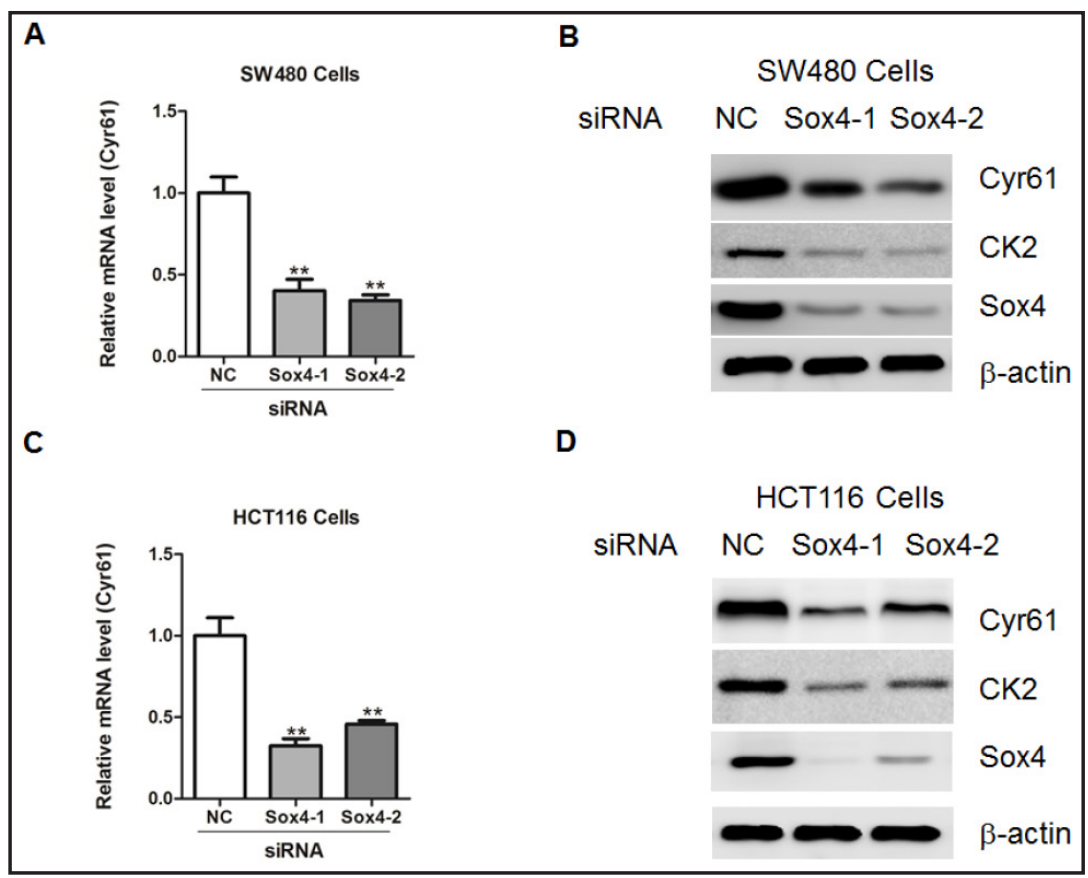
rols (NC) for $24 \mathrm{hr}$. Total AKT and P65
Fig. 3. Sox 4 affects AKT and P55 activation in colon cancer cells. (A-B) Western blot analysis of phosphorylated AKT and P65 levels in SW480 (A) or HCT116 (B) cells transfected with Sox4 expression plasmids or empty vector (EV) for $24 \mathrm{hr}$. Total AKT and P65 contents were set as loading controls. (C-D) Western blot analysis of phosphorylated AKT and P65 levels in SW480 (C) or HCT116 (D) cells transfected with siRNA oligos targeting Sox4 or negative contcontents were set as loading controls. (E)The cell proliferative potential (BrdU) was determined in SW480 cells. Cells were pre-transfected with siRNA oligos targeting Cyr61 or NC for $24 \mathrm{hr}$, and then transfected with Sox4 expression plasmids or EV for another $24 \mathrm{hr}$. (F) Protein contents of Cyr61 were determined by Western blot in SW480 cells transfected with siRNA oligos targeting Cyr61 or NC.
E

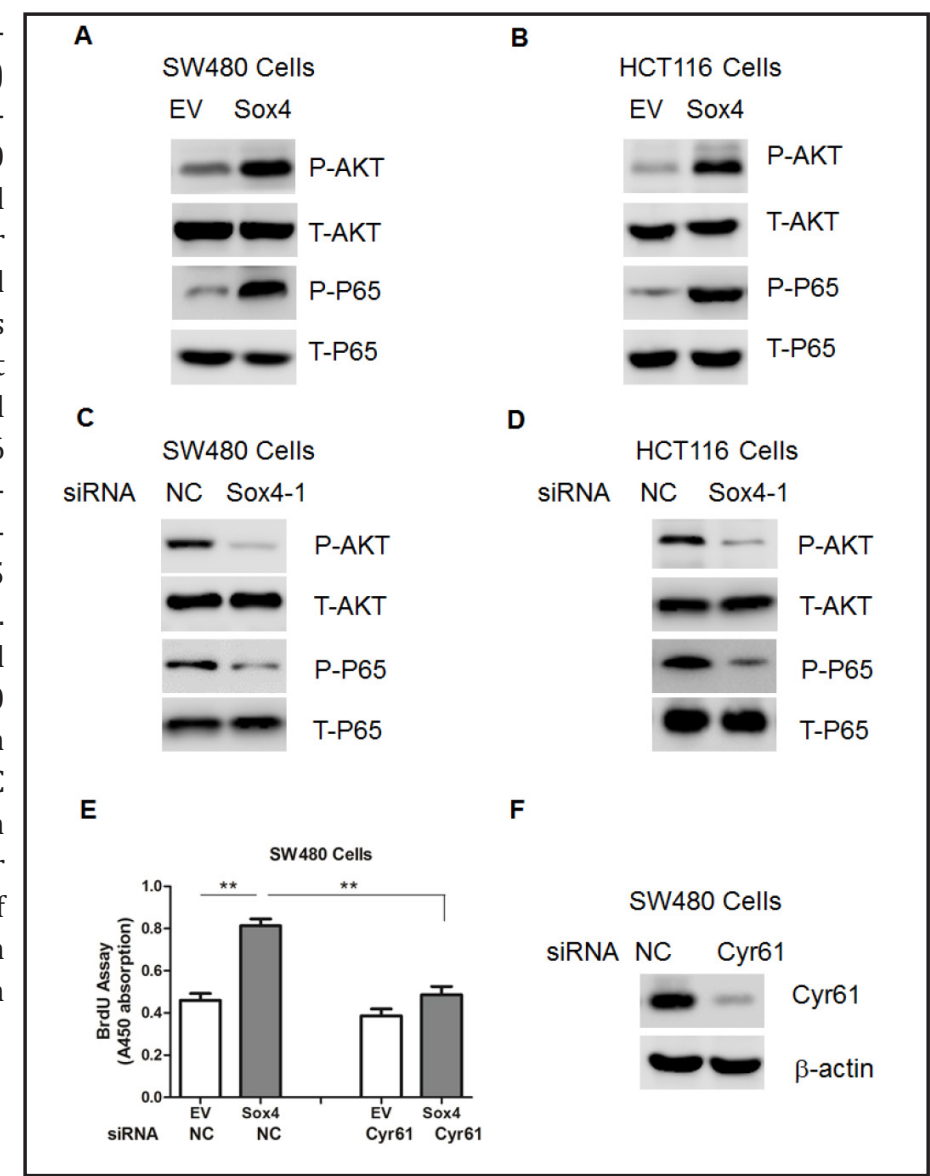

the transcriptional start site (Fig. 5A). Therefore, we transfected SW480 cells with a reporter vector encoding Luciferase under control of the Cyr61 promoter (PGL4-WT-Cyr61) (Fig. 5B). Concurrent expression of Sox4 with the Cyr61 reporter construct increased Cyr61 promoter activity (Fig. 5B), which was abrogated by mutation of the Sox4 DNA-binding site in the 
Fig. 4. Sox4 regulates Cyr61 expression in vivo. (A-B) SW480 cells overexpressing Sox4 or empty vector (EV) were injected into nude mice ( $n=5$ for each group) and followed up for tumorigenesis. Representative pictures (A) and tumor weights (B) were taken 4 weeks after injection. (C-D) mRNA and protein levels of Cyr61 were determined by realtime PCR (C) and western blot (D) in tumors overexpressing Sox 4 or empty vector (EV).

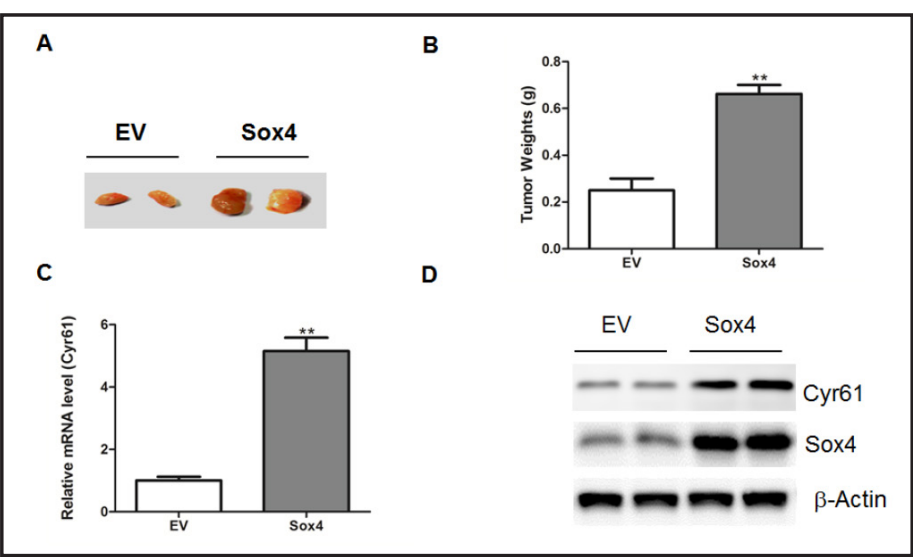

A

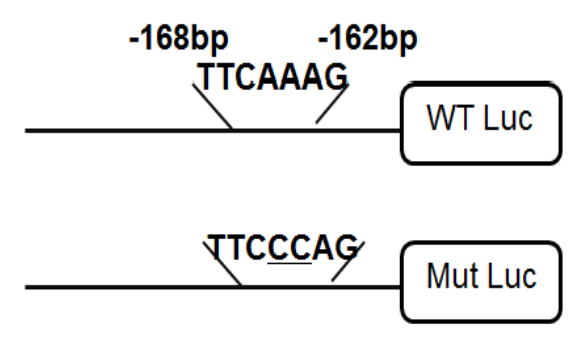

C

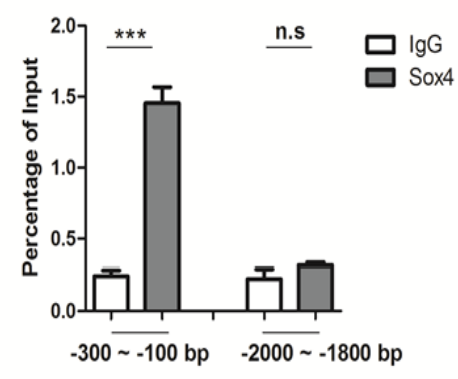

B

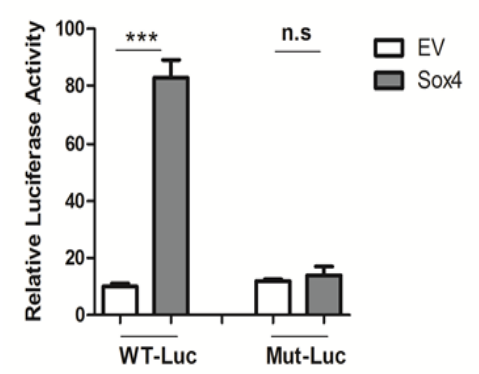

D

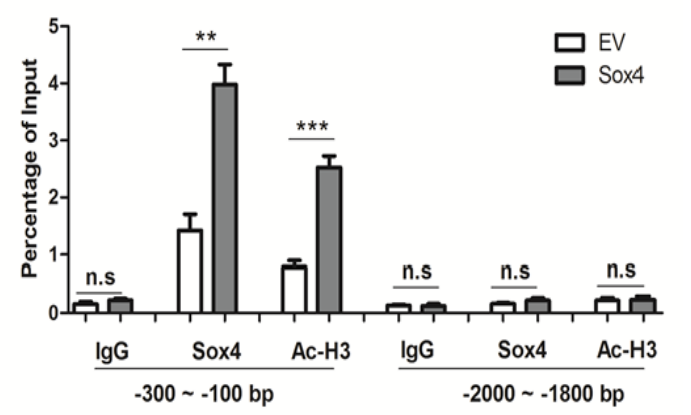

Fig. 5. Sox4 is a direct transcriptional activator of Cyr61. (A) The human Cyr61 promoter constructs containing a potential Sox 4 binding motif ( -168 bp to -162 bp). (B) The transcriptional activity of the Cyr61 promoter. SW480 cells were co-transfected with the indicated plasmids for $24 \mathrm{~h}$, and the luciferase activity was measured. The wild-type Cyr61 promoter (WT-Luc) was up-regulated by Sox4, whereas the point mutant promoter (Mut-Luc) abolished the Sox4-mediated transcriptional activity. (C) ChIP assays of Sox4 and its binding motif. Two antibodies (anti-IgG and - Sox4) were used in the ChIP assays using SW480 cells. The promoter region from -2000 to $-1800 \mathrm{bp}$ was set as a negative control. Real-time PCR was performed to quantify the binding activity. (D) ChIP assays with the indicated antibodies using nuclear lysates in SW480 cells overexpressing Sox 4 or EV and quantified by real-time PCR (right panel). Ac-H3, acetylhistone H3. ** $\mathrm{p}<0.01,{ }^{* * *} \mathrm{p}<0.001$.

Cyr61 promoter (Fig. 5B). In addition, our chromatin immunoprecipitation (ChIP) assays also confirmed that Sox 4 could directly bind with this region, but not the distal region of Cyr61 promoter (Fig. 5C). Consistently, Sox4 overexpression recruited much more acetylated histone $\mathrm{H} 3$ to the Cyr61 proximal promoter, a marker of actively transcribed genes (Fig. 5D). 


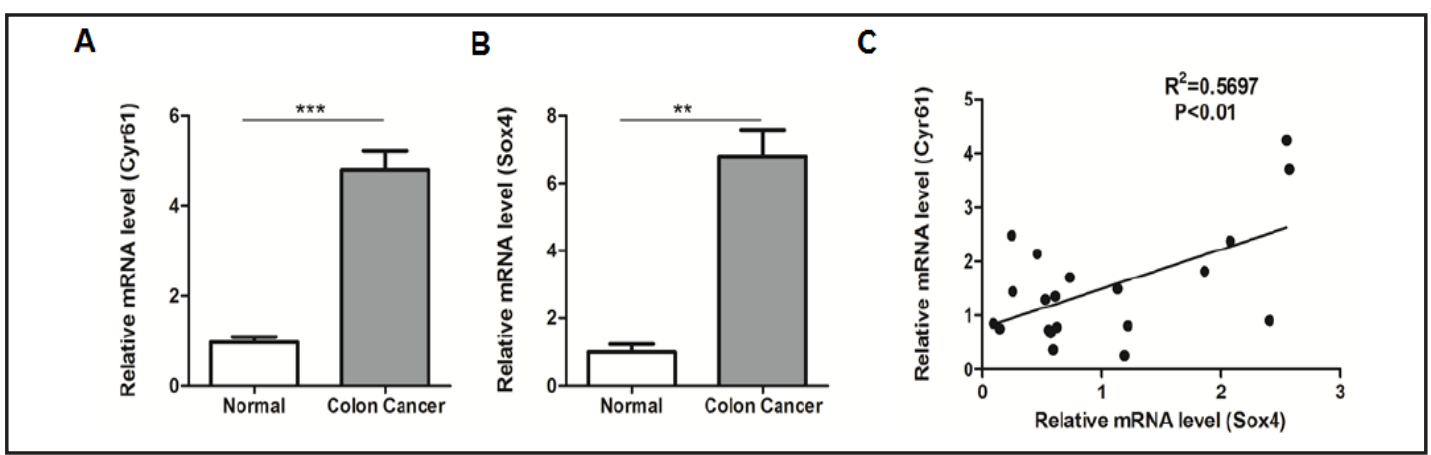

Fig. 6. Up-regulation of Cyr61 and Sox4 in human colon cancer tissues. (A-B) mRNA levels of Cyr61 and Sox4 in human colon cancer tissues and adjacent normal tissues. (C) Pearson correlation analysis of mRNA levels of Sox 4 and Cyr61 in human subjects. ${ }^{* * *} \mathrm{p}<0.001$.

Cyr61 expression levels were increased in colon cancer tissues

Finally, we examined whether Cyr61 was differentially expressed in human colon cancer tissues. Our results revealed that mRNA levels of Cyr61 and Sox4 were significantly increased in cancer tissues, compared with pair-matched adjacent normal tissues (Fig. 6A6B). Moreover, a positive correlation between mRNA levels of Sox4 and Cyr61 was observed (Fig. 6C), further indicating the transcriptional regulation of Cyr61 by Sox 4 .

\section{Discussion}

In the present study, we demonstrate that Sox4 up-regulates Cyr61 expression using two human colon cells. Sox 4 overexpression promotes while its silencing reduced Cyr61 mRNA and protein levels. At the molecular level, we identified a potential Sox 4 binding site in the proximal region of Cyr61 gene promoter. However, whether Sox 4 could regulate Cyr61 expression in other cancer cells remains to be defined.

Previous studies have shown that Sox 4 could directly control expression levels of several key cellular regulators in prostate cancer, such as EGFR, HSP70, Tenascin C, Frizzled-5, Patched-1, and Delta-like 1 [16]. Besides, Sox4 could interact with P53 to inhibit P53induced cell apoptosis in hepatocellular carcinoma [17]. Moreover, Sox4 promotes epithelialmesenchymal transition in breast cancer cells by controlling Ezh2 expression and epigenetic reprogramming [18]. In colon cancer, Sox 4 was shown to regulate $\beta$-catenin/T-cell factor activity and act as an agonist of Wnt signaling, through induction of casein kinase $2[19,20]$. Here, we found that Sox 4 overexpression could enhance signaling pathways of Akt and NF$\mathrm{kb} / \mathrm{P} 65$. Therefore, together with these findings, our data suggest a diverse role for Sox 4 in tumorigenesis.

Up-regulation of Cyr61 has been observed in many human tumors, including colon cancers [21,22], suggesting its important roles in tumor initiation or development. However, its molecular determinants remain largely unknown. Recent studies indicate that Cyr61 expression was negatively regulated by MicroRNA-100 and MicroRNA-22 in osteosarcoma and rheumatoid arthritis, respectively [23, 24]. Given that Sox4 expression is usually abnormally expressed in multiple cancers, our results suggest a novel mechanism for the up-regulation of Cyr61 in cancers.

In summary, our results add new insights into how Sox 4 could regulate Cyr61 expression in colon cancer cells. The molecular network involving Sox4 and Cyr61 might provide a potential therapeutic option for human cancers in the future.

\section{Disclosure Statement}

None 
Wu/Zhu/Zhang: Sox4 Up-Regulates Cyr61

\section{References}

-1 1 Kuipers EJ, Rösch T, Bretthauer M: Colorectal cancer screening-- optimizing current strategies and new directions. Nat Rev Clin Oncol 2013;10:130-142.

2 Harris TJ, McCormick F: The molecular pathology of cancer. Nat Rev Clin Oncol 2010;7:251-265.

- 3 Tenesa A, Dunlop MG: New insights into the aetiology of colorectal cancer from genome-wide association studies. Nat Rev Genet 2009;10:353-358.

-4 Penzo-Méndez AI: Critical roles for SoxC transcription factors in development and cancer. Int J Biochem Cell Biol 2010;42:425-428.

5 Jafarnejad SM1, Ardekani GS, Ghaffari M, Li G: Pleiotropic function of SRY-related HMG box transcription factor 4 in regulation of tumorigenesis. Cell Mol Life Sci. 2013;70:2677-2696.

6 Schilham MW, Oosterwegel MA, Moerer P, Ya J, de Boer PA, van de Wetering M, Verbeek S, Lamers WH, Kruisbeek AM, Cumano A, Clevers H: Defects in cardiac outflow tract formation and pro-B-lymphocyte expansion in mice lacking Sox-4. Nature 1996;380:711-714.

7 Cheung M, bu-Elmagd M, Clevers H, Scotting PJ: Roles of Sox4 in central nervous system development. Brain Res Mol Brain Res 2000;79:180-191.

8 Wang L, Zhang J, Yang X, Chang YW, Qi M, Zhou Z, Zhang J, Han B: SOX4 is associated with poor prognosis in prostate cancer and promotes epithelial-mesenchymal transition in vitro. Prostate Cancer Prostatic Dis 2013;16:301-307.

-9 Gunes S, Yegin Z, Sullu Y, Buyukalpelli R, Bagci H. SOX4 expression levels in urothelial bladder carcinoma. Pathol Res Pract. 2011;207: 423-427.

10 Castillo SD, Matheu A, Mariani N, Carretero J, Lopez-Rios F, Lovell-Badge R, Sanchez-Cespedes M: Novel transcriptional targets of the SRY-HMG box transcription factor SOX4 link its expression to the development of small cell lung cancer. Cancer Res 2012;72:176-186.

11 Vervoort SJ, van Boxtel R, Coffer PJ: The role of SRY-related HMG box transcription factor 4 (SOX4) in tumorigenesis and metastasis: friend or foe? Oncogene 2013;32:3397-3409.

$\checkmark 12$ Lin CM, Fang CL, Hseu YC, Chen CL, Wang JW, Hsu SL, Tu MD, Hung ST, Tai C, Uen YH, Lin KY: Clinical and prognostic implications of transcription factor sOX4 in patients with colon cancer. PLoS One 2013;8:e67128.

13 Lee KB, Byun HJ, Park SH, Park CY, Lee SH, Rho SB: CYR61 controls p53 and NF-кB expression through PI3K/Akt/mTOR pathways in carboplatin- induced ovarian cancer cells. Cancer Lett 2012;315:86-95.

14 Goodwin CR, Lal B, Zhou X, Ho S, Xia S, Taeger A, Murray J, Laterra J: Cyr61 mediates hepatocyte growth factor-dependent tumor cell growth, migration, and Akt activation. Cancer Res. 2010;70:2932-2941.

15 Lu Y, Xiong X, Wang X, Zhang Z, Li J, Shi G, Yang J, Zhang H, Ning G, Li X. Yin Yang 1 promotes hepatic gluconeogenesis through upregulation of glucocorticoid receptor. Diabetes 2013;62:1064-1073.

16 Scharer CD, McCabe CD, Ali-Seyed M, Berger MF, Bulyk ML, Moreno CS: Genome-wide promoter analysis of the SOX4 transcriptional network in prostate cancer cells. Cancer Res 2009;69:709-717.

17 Zhang J, Liang Q Lei Y, Yao M, Li L, Gao X, Feng J, Zhang Y, Gao H, Liu DX, Lu J, Huang B: SOX4 induces epithelial-mesenchymal transition and contributes to breast cancer progression. Cancer Res 2012;72:4597-4608.

18 Hur W, Rhim H, Jung CK, Kim JD, Bae SH, Jang JW, Yang JM, Oh ST, Kim DG, Wang HJ, Lee SB, Yoon SK: SOX4 overexpression regulates the p53-mediated apoptosis in hepatocellular carcinoma: clinical implication and functional analysis in vitro. Carcinogenesis 2010;31:1298-1307.

19 Lee AK, Ahn SG, Yoon JH, Kim SA: Sox4 stimulates ß-catenin activity through induction of CK2. Oncol Rep 2011;25:559-565.

20 Sinner D, Kordich JJ, Spence JR, Opoka R, Rankin S, Lin SC, Jonatan D, Zorn AM, Wells JM: Sox17 and Sox4 differentially regulate beta-catenin/ T-cell factor activity and proliferation of colon carcinoma cells. Mol Cell Biol 2007;27:7802-7815.

21 Lin J, Huo R, Wang L, Zhou Z, Sun Y, Shen B, Wang R, Li N: A novel anti-Cyr61 antibody inhibits breast cancer growth and metastasis in vivo. Cancer Immunol Immunother 2012;61:677-687.

22 Ladwa R, Pringle H, Kumar R, West K: Expression of CTGF and Cyr61 in colorectal cancer. J Clin Pathol 2011;64:58-64.

23 Huang J, Gao K, Lin J, Wang Q: MicroRNA-100 inhibits osteosarcoma cell proliferation by targeting Cyr61. Tumour Biol 2014;35:1095-1100.

24 Lin J, Huo R, Xiao L, Zhu X, Xie J, Sun S, He Y, Zhang J, Sun Y, Zhou Z, Wu P, Shen B, Li D, Li N:. A novel p53/ microRNA-22/Cyr61 axis in synovial cells regulates inflammation in rheumatoid arthritis. Arthritis Rheumatol 2014;66:49-59. 